Keywords: Group psychoeducation, Schizophrenia, Forensic setting, Knowledge, Awareness of illness, Attitudes toward psychiatric treatment and medication.

\title{
Group psychoeducation for forensic and dangerous non-forensic long-term patients with schizophrenia. A pilot study
}

\author{
Kati Aho-Mustonen, M.A. (Psych)* \\ Raili Miettinen, M.A. (Psych) ${ }^{\star \star}$ \\ Hanna Koivisto, M.A. (Psych) ${ }^{\star \star}$ \\ Tero Timonen, PhD. (Psych)* \\ Hannu Räty, PhD. (Psych) ${ }^{\star}$ \\ * University of Joensuu, Department of \\ Psychology, Joensuu \\ ** University of Kuopio, Department of Forensic \\ Psychiatry, Niuvanniemi Hospital, Kuopio \\ FINLAND
}

\begin{abstract}
Background and Objectives: The effectiveness of patient psychoeducation has only rarely been studied among forensic populations. This paper examines the outcomes of an eight-time psychoeducational group comprised of schizophrenic patients in a forensic hospital setting in Finland.

Methods: The intervention group contained forensic or dangerous non-forensic longterm patients with schizophrenia. Treatment outcomes were compared between the intervention group $(n=7)$ and a matched treatment-as-usual control group $(n=8)$. The group provided information about schizophrenia and its treatment and cognitive-behavioural elements were used to enhance patients' learning and coping. The results were obtained from a knowledge of schizophrenia, awareness of mental disorder, attitudes toward psychiatric treatment and medication, and depression.

Results: The results of this pilot study suggest that even severely ill patients were able to gain improved knowledge about their illness, and psychoeducation also had positive impact on their awareness of the illness. The results showed no significant changes in attitudes toward psychiatric treatment or medication, or depressive symptoms. No significant changes in any measures were found in the control group.

Conclusions: Despite our positive results further research with larger samples is needed to discover the effectiveness of patient education as a component of the comprehensive treatment of forensic patients with schizophrenia.
\end{abstract}

Received 9 November 2007

Revised 11 June 2008

Accepted 16 June 2008 


\section{Introduction}

The treatment of forensic schizophrenic patients poses many challenges as a result of the severity of the patients' illness, the often persistent symptomalogy, and many related problems, such as aggressive behaviour. Also comorbid problems, such as substance abuse and personality disorders, are common among forensic patients. Research has shown that patients with comorbid mental disorders are more resistant to treatment, and are subject to a more chronic course and a poorer prognosis of their illness ${ }^{1}$. Forensic schizophrenic patients often need long-term hospitalization, and before discharge from the hospital becomes possible, clinical forensic psychiatry needs to ascertain that the patient has sufficient insight into illness, has a developed adherence with medication, and is also capable of, and motivated to maintain, long-term use of community psychiatric services ${ }^{2}$.

Psychoeducation is considered an important and ethically indispensable part of the comprehensive treatment of schizophrenia ${ }^{3,4}$. Teaching a patient about his/her illness can increase the patient's coping skills and understanding of the early signs of psychosis and the stressful elements anticipated in and related to psychotic episodes. The main principal of psychoeducation is that everyone has the right to receive information about their illness and its treatment in order for them to take a more active role in relation to the disease and its treatment ${ }^{5,6}$.

Psychoeducation has shown positive effects in relapse prevention ${ }^{7,8}$. Most studies of psychoeducation for patients only have demonstrated that knowledge and adherence can be improved by means of educational interventions ${ }^{9}$. Studies using more didactic formats have influenced improve- ments in general understanding of schizophrenia and treatment ${ }^{10-12}$. Evidence exists that even comparatively brief interventions can achieve significant improvements in patients with chronic psychiatric disorders ${ }^{10,12-14}$. A recently published meta-analysis conducted by Lincoln, Wilhelm \& Nestoriuc ${ }^{15}$ suggests, however, that psychoeducation for patients only can not be considered effective. Psychoeducation is nowadays commonly provided within forensic settings ${ }^{16}$, but the systematic review of structured group interventions with mentally disordered offenders made by Duncan et al. ${ }^{17}$ shows that the effectiveness of patient psychoeducation among forensic schizophrenia patients has been studied only by the naturalistic pilot study of Jennings et al. ${ }^{18}$, who found positive a impact of brief patient group psychoeducation on knowledge, attitudes toward medication, and insight.

The aim of the present study has been to analyze the effectiveness of an eight-session educational intervention with long-term forensic and hard-to-treat non-forensic schizophrenia patients. The aim was to assess and compare treatment outcomes between the psychoeducation group and the matched control group in relation to their knowledge of schizophrenia. Changes in awareness of mental disorder, and attitudes toward psychiatric treatment and medication were also measured. The effects of this intervention on participants' depressive symptoms were also examined.

\section{Method}

\section{Service setting and participants}

Ethical approval for the research was obtained from the research ethics committee 
of the Kuopio University Hospital. The study group consisted of male mentally ill offenders and hard-to-treat dangerous patients at the Niuvanniemi forensic hospital in Kuopio, Finland. The trial was conducted during the summer of 2001. Patients at the Niuvanniemi hospital have usually experienced several previous hospitalizations: forensic patients an average of 9 times, dangerous and hardto-treat patients an average of 11 times. At the end of December 2001, the average duration of treatment undergone by the forensic patients was 7 years 10 months, by the nonforensic patients 4 years 2 months.

The intervention was conducted as an intervention versus control group setting. In the beginning of selection of the intervention group staff at the ward evaluated the patients who met the DSM-IV criteria ${ }^{19}$ for a primary diagnosis of schizophrenia. Patients with organic brain syndrome were excluded from the study. All of the patients were currently taking neuroleptic medication. Patients whose psychic condition was sufficiently stable were then recommended for a psychological interview, where information about the study group was given to each patient. Eight of the 11 patients interviewed signed written contracts of informed consent indicating their willingness to participate. One participant had to leave the group in the early phase of the intervention owing to a move to another ward, and was eventually excluded from the statistical analyses. Thus, the final sample in the intervention group consisted of seven patients, of whom four were forensic patients and the remaining three were hard-to-treat dangerous patients. Five patients in the intervention group had a comorbid diagnosis of substance abuse (alcohol or psychoactive drugs), and three participants had a comorbid personality disorder.

The control group, consisting of 8 male patients from another ward, was matched with the intervention group in terms of age, main and secondary diagnoses, current score on the Global Assessment of Functioning (GAF) Scale, and patient statuses. Half of the group, 4 patients, were forensic patients, and 4 were hard-to-treat dangerous patients. Six patients in the control group had a comorbid substance abuse disorder, and three patients were diagnosed as suffering from a comorbid personality disorder.

\section{Measures}

\section{Knowledge of Schizophrenia}

"Knowledge of Schizophrenia" is a 23item multiple-choice questionnaire adapted by the first author from the 25-item "Schizophrenia Questionnaire" of Ascher-Svanum and Krause ${ }^{20}$, subsequently referred to as the KASQ (The Knowledge About Schizophrenia Questionnaire). The questionnaire covers the illness-related topics concerned in the education programme. The psychometric properties of the KASQ have been investigated, and information about the construct validity of the questionnaire and its reliability has been provided ${ }^{21}$. In the version used in this study the items were translated into Finnish and the two items dealing with legal issues (items 20 and 21) were excluded. An estimate of the internal consistency of the Finnish version was calculated in this sample and an adequate coefficient alpha (KR20) was found ( $\alpha=0.74)$. The maximum score is 23 , indicating a high level of knowledge about schizophrenia.

\section{Awareness of illness and attitudes toward psychiatric treatment and medication}

Awareness of illness and attitudes toward psychiatric treatment and medication were 
assessed by means of a 25-item questionnaire developed by the first author. We wanted to develop a self-report instrument that would be easily administered and easy for patients to understand. The questionnaire covers important topics in forensic psychiatry related to the patients' subjective evaluations of their attitudes toward treatment and medication, and their understanding of their illness. Their subjective opinions about their quality of life were also assessed using this selfreport instrument, but these will not be reported here. Patients' awareness of illness was assessed by means of items including statements such as "I have symptoms of psychiatric illness". Attitudes to medication were assessed with items such as: "I have benefited from the medication prescribed to me", and attitudes toward psychiatric treatment with items such as "I usually agree with the nursing staff over the issues connected with my treatment". The questionnaire was developed to be as easy as possible to answer due to the cognitive problems of the participants, thus enabling them to understand the instructions and answer the questionnaire independently. Patients rated the items on a three-point Likert scale ranging from $1=$ "totally disagree" to $3=$ "totally agree". The alpha coefficients for these scales were calculated in this sample as: awareness of illness $(\alpha=0.73)$, attitude toward psychiatric treatment $(\alpha=0.80)$, and attitude toward medication $(\alpha=0.86)$, demonstrating that the scales have sufficient internal consistency. The maximum mean score for each scale is 3 , indicating positive attitudes toward medication and treatment and good awareness of illness.

\section{Depression}

The level of self-reported depressive symptoms was assessed using the 21-item Beck Depression Inventory ${ }^{22}$. The maximum score of the inventory is 63 , indicating severe depression $^{23}$.

\section{Statistical analyses}

The statistical analyses were carried out using non-parametric tests because of the small sample sizes. Differences in baseline scores of the assessments between the groups, and differences in mean change of the scores over time between groups were analysed by the Mann-Whitney $U$ test. The Mann-Whitney $U$ analyses were also conducted across the demographic variables in the intervention and control groups. The changes over time within the groups were analysed by the Wilcoxon signed rank-test.

\section{Procedure}

The psychoeducational program used in this study was adapted and modified from Ascher-Svanum and Krause's "Psychoeducational Groups for Patients with Schizophrenia", based on the stress-vulnerability model of schizophrenia ${ }^{20}$. The modifications included putting special emphasis on topics essential in the treatment of forensic patients (e.g. alcohol and drug abuse) and updating the information about schizophrenia. The didactic information followed the Finnish Schizophrenia practice guideline ${ }^{24}$. In addition to providing didactic information the intervention aimed to enhance participants' coping with the illness, and program contained cognitive-behavioural elements. Participants were, for example, assigned homework between group sessions. A "normalizing" rationale to explain symptom emergence in schizophrenia was used ${ }^{25}$. 
The intervention group participated in an eight-session intervention once a week and the sessions were 45-60 minutes long. The sessions were conducted by two psychologists (the first and second authors of this article). The patients' possible cognitive deficits were taken into account, and each session included educational techniques designed to maintain their attention and to enhance learning. The clinicians encouraged the patients to articulate their own opinions and experiences. Topics covered in the group sessions included: orientation, definition of schizophrenia and common symptoms, diagnosis and etiology of schizophrenia, course of illness, outcome of schizophrenia and warning signs of relapse, causes of schizophrenia, the stress-vulnerability model and the influence of stress, substance abuse in schizophrenia, antipsychotic medication, psychosocial treatment of schizophrenia, and legal issues. The intervention group utilized a leader manual.

The control group received the usual treatment provided in forensic psychiatry, i.e. psychopharmacological treatment, the usual ward activities, and psychosocial rehabilitation efforts.

\section{Results}

Evaluations for the whole sample were administered by the third author (HK), as an independent expert, before the intervention group attended the first session, and after the last group session.

\section{Diagnostic and demographic variables}

Mann-Whitney $U$ analyses were conducted across the demographic variables in the inter- vention and control groups. The analyses revealed no significant differences between the groups with regard to their age (intervention group mean age 40.4, SD 6.24, control group 38.4 , $\mathrm{SD} 7.65, p=0.63$ ) or their assessed general functioning (intervention group mean GAF 36.1, SD 7.90, control group 39.4, SD 5.78, $p=0.26$ ).

\section{Self-report instruments}

The results were obtained from five main areas: knowledge of schizophrenia, awareness of illness, attitudes toward psychiatric treatment and medication, and depression. A summary of the main results is shown in Table I.

\section{Treatment outcome}

\section{Knowledge of schizophrenia}

The baseline knowledge of schizophrenia was higher in the control group, but the difference between the group participants and the controls was not statistically significant ( $U=17.00, p=0.23$ ). The intervention group showed improved knowledge about schizophrenia after the intervention $(Z=$ $-2.371, p=0.02)$. No change was found in the control group $(Z=-0.38, p=1.00)$. The change in mean knowledge scores between the groups was statistically significant $(U=$ $1.50, p<0.01)$.

\section{Awareness of illness and attitudes toward psychiatric treatment and medication}

At the baseline there was no significant difference between the intervention partici- 


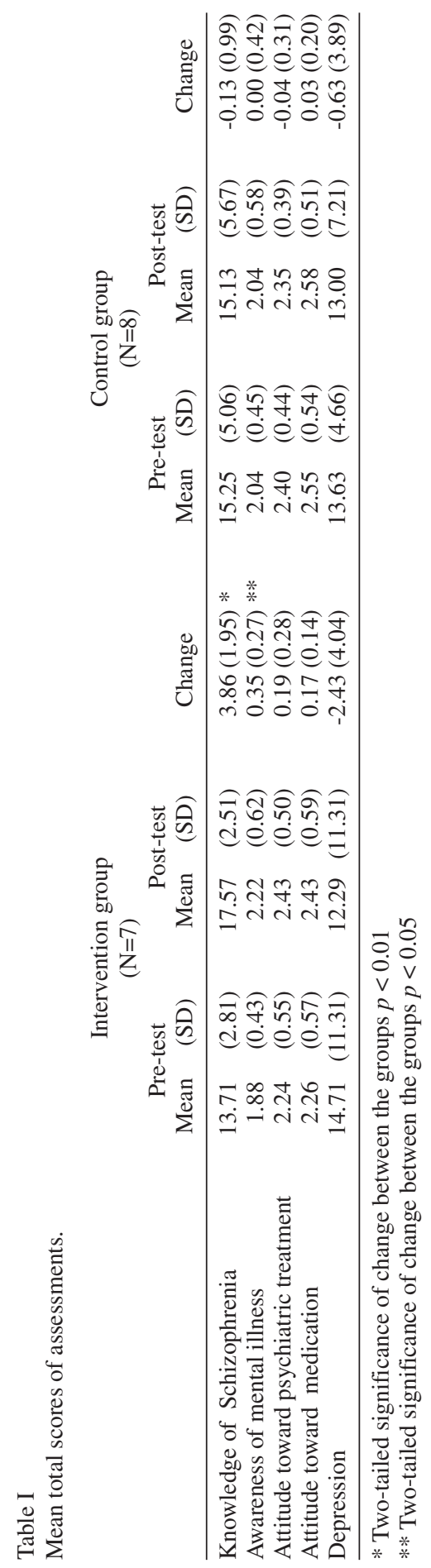

pants and the controls in terms of their awareness of illness, although the scores in the control group were somewhat higher ( $U=27.00, p=0.96)$. Intervention group members showed improved awareness of their illness after the intervention $(Z=-2.21$, $p=0.03)$. No change was found in the control group $(Z=-0.34, p=0.80)$. The change in scores between the groups was statistically significant $(U=10.00, p=0.04)$.

There was no significant difference between the group participants and the controls at the baseline in terms of their attitude toward psychiatric treatment $(U=24.00, p=$ $0.69)$. At the post-group intervention stage the intervention group showed an improved treatment attitude score $(Z=-1.63, p=0.19)$. No significant change was found in the control group $(Z=-0.51, p=0.65)$. The change scores concerning attitude toward psychiatric treatment between the groups remained statistically insignificant $(U=17.50, p=$ $0.23)$.

At the baseline the patients' attitudes toward their medication were relatively positive in both groups $(U=18.50, p=0.28)$. After intervention there was a trend of improved attitudes toward medication in the intervention group $(Z=-2.121, p=0.06)$. No change was found in the control group $(Z=-0.38, p=1.00)$. The change in medication attitude scores between the groups still remained statistically insignificant $(U=14.50$, $p=0.12$ ).

\section{Depression}

There was no significant difference between the intervention participants and the controls at the baseline in terms of depression assessed by the Beck Depression Inventory ( $U=25.50, p=0.78)$. After the intervention the intervention group showed 
some improvement in terms of reduced depressive thoughts $(Z=-1.53, p=0.17)$. No significant change in depression scores was found in the control group $(Z=-0.42$, $p=0.74)$. The change in depression scores between the groups was not statistically significant $(U=20.00, p=0.40)$.

\section{Discussion}

Despite the fact that the patients in our sample had undergone several earlier hospitalizations and suffered a long period of illness, they displayed a clear need for information about their illness and treatment, and their baseline understanding of their illness was low. This has also been noted in earlier studies involving schizophrenic inpatient groups ${ }^{26}$. The results of this pilot study indicate that, in comparison with the control group, the study group's knowledge of schizophrenia increased during the intervention. This finding is consistent with the results obtained from previous psychoeducational interventions for patients with schizophrenia among non-forensic and forensic patients, indicating that, even in the context of psychoeducational groups with a quite didactic group format, improvements in understanding can be made ${ }^{10-12,18}$. The present study suggests that a certain level of knowledge gains is possible even amongst the most severely ill patients suffering from cognitive deficits and disturbances, although increase in knowledge scores was lowest among patients with the most severe psychotic symptomalogy.

No statistically significant impact of the psychoeducation group on the patients' attitudes toward psychiatric treatment or medication was found in this study. Positive findings were found, however, in regard to patients' awareness of their illness, and the result is consistent with the previous results of Jennings et al. ${ }^{18}$ concerning psychoeducation for mentally ill offenders. This result is encouraging because awareness of illness is an important aspect of insight, and a lack of insight is considered a common problem in relapse prevention ${ }^{27}$.

The effects of psychoeducational interventions on the depressive symptoms of the participants have to be considered because sometimes enhanced insight may be associated with increased suicidal ideation ${ }^{28}$. In this study the participants' depressive symptoms were assessed before and after intervention. The results showed no significant changes in these scores for the intervention group, and it can be concluded that such psychoeducational intervention does not seem to have a significant impact in terms of the participants' depressive symptoms.

The acceptance of the group was high. All of the participants who started the group stayed in the group. The results of this study are encouraging because forensic psychiatric patients are often committed to hospital care against their will, and consequently their motivation to undergo treatment is lower than usual.

A strength of this study was its attempt to apply and evaluate a brief psychoeducational programme on forensic or hard-to-treat schizophrenic patients suffering from severe mental illness. Comorbidity amongst forensic patients is high and constitutes a major challenge to treatment, but patients with a history of comorbid substance abuse or non-adherence or treatment resistance have, however, often been excluded from clinical studies ${ }^{29}$. The results of this pilot study suggest that patients with comorbid problems can also benefit from a psychoeducational group and suggests that psychoeducational group inter- 
vention can constitute a useful component in a comprehensive approach to the treatment of long-term forensic schizophrenia patients, combined with antipsychotic medication and other rehabilitation methods.

The limitations of this study include the small group sizes and its lack of a randomized design, both of which prevent the generalization of the results in terms of larger populations. The small sample sizes also permitted only a non-parametric test for the data analyses. Limitation of this study is also the use of unstandardized measure of insight. Our study also lacks a follow-up period, and hence we cannot tell whether the changes discovered in the patients' knowledge and awareness of illness were capable of enduring. Hence, the long-term effects remain unknown. Without booster sessions to reinforce and consolidate gains, long-term effects may not be sustainable $^{30}$. Further research with larger samples and a randomized study design with an adequate follow-up period is therefore needed to examine the efficacy and long-term effectiveness of patient education among forensic populations.

\section{References}

1. Newman DL, Moffitt TE, Caspi A, Silva PA. Comorbid mental disorders: implications for treatment and sample selection. J Abnorm Psychol 1998; 107: 305-311.

2. Tiihonen J. Oikeuspsykiatria. In: Lönnqvist J, Heikkinen M, Henriksson M, Marttunen M, Partonen T, eds. Psykiatria ( $5^{\text {th }}$ revised edition). Jyväskylä: Gummerus; 2007 p. $650-667$.

3. Falloon IRH, Held T, Roncone R, Coverdale JH, Laidlaw TM. Optimal treatment strategies to enhance recovery from schizophrenia. Aust N Z J Psychiatry 1998; 32: 43-49.

4. Bäuml J, Froböse T, Kraemer S, Rentrop M, PitschelWalz G. Psychoeducation: A basic psychotherapeutic inter- vention for patients with schizophrenia and their families. Schizophr Bull 2006; 32(S1): S1-S9.

5. McGlashan TH. What has become of the psychotherapy of schizophrenia? Acta Psychiatr Scand Suppl 1994; 90(384): 147-152.

6. McGorry PD, Edwards J. Early psychosis training pack. Macclesfield, Cheshire: Gardiner-Caldwell Communications Ltd; 1997.

7. Pekkala E, Merinder L. Psychoeducation for schizophrenia. Cochrane database Syst Rev 2002; (2): CD002831.

8. Rummel-Kluge C, Kissling, W. Psychoeducation in schizophrenia: new developments and approaches in the field. Current Opinion in Psychiatry, 2008; 21: 168-172.

9. Merinder LB. Patient education in schizophrenia: a review. Acta Psychiatr Scand 2000; 102: 98-106.

10. Goldman CR, Quinn FL. Effects of a patient education program in the treatment of schizophrenia. Hosp Community Psychiatry 1988; 39(3): 282-286.

11. Smith J, Birchwood M. Informing people with schizophrenia about their illness: the effect of residual symptoms. J Ment Health 1992; 1: 61-70.

12. Merinder L-B, Viuff AG, Laugesen HD, Clemmensen K, Misfelt S, Espensen B. Patient and relative education in community psychiatry: a randomized controlled trial regarding its effectiveness. Soc Psychiatry Psychiatr Epidemiol 1999; 34: 287-294.

13. Hornung WP, Kieserg A, Feldmann R, Buchkremer G. Psychoeducational training for schizophrenic patients: backround, procedure and empirical findings. Patient Educ Couns 1996; 29: 257-268.

14. Kelly GR, Scott JE. Medication compliance and health education among outpatients with chronic mental disorders. Med Care 1990; 28(12): 1181-1197.

15. Lincoln TM, Wilhelm K, Nestoriuc Y. Effectiveness of psychoeducation for relapse, symptoms, knowledge, adherence and functioning in psychotic disorders: A metaanalysis. Schizophrenia Res 2007; 96: 232-245.

16. Walker H. Using psychosocial interventions within a high-security hospital. Nurs Times 2004; 100(31): 36-39.

17. Duncan EAS, Nicol MM, Ager A, Dalgleish L. A systematic review of structured group interventions with mentally disordered offenders. Crim Beh Ment Health 2006; 16: 217-241.

18. Jennings L, Harris B, Gregoire J, Merrin J, Peyton J, Bray L. The effect of a psycho-educational programme on knowledge of illness, insight and attitudes towards medication. Br J Forensic Pract 2002; 4(2): 3-10. 
19. American Psychiatric Association. Diagnostic and Statistical Manual of Mental Disorders ( $4^{\text {th }}$ edition). Washington, DC: APA; 1994.

20. Ascher-Svanum H, Krause A. Psychoeducational groups for patients with schizophrenia. Gaithersburg, MD: Aspen Publishers; 1991.

21. Ascher-Svanum H. Development and validation of a measure of patients' knowledge about schizophrenia. Psychiatr Serv 1999;50:561-563.

22. Beck AT, Ward $\mathrm{CH}$, Mendelson M, Mock J, Erbaugh J. An inventory for measuring depression. Arch Gen Psychiatry 1961; 4: 561-571.

23. Beck AT, Steer RA, Garbin MG. Psychometric properties of the Beck Depression Inventory: Twenty-five years of evaluation. Clin Psychol Rev 1988; 8: 77-100.

24. Salokangas, RKR, ed. Skitsofrenia. Käypä HoitoSuositus. Duodecim 2001; 117: 2640-2657.

25. Kingdon DG, Turkington D. Cognitive-Behavioural Therapy of schizophrenia. Hove: Lawrence Erlbaum; 1994.

26. Macpherson R, Jerrom B, Hughes A. A controlled study of education about drug treatment in schizophrenia. Br J Psychiatry 1996; 168: 709-717.
27. Birchwood M, Spencer E, McGovern D. Schizophrenia: early warning signs. Adv Psychiatr Treat 2000; 6: 93-101.

28. Cunningham Owens DG, Carroll A, Fattah S, Clyde Z, Coffey I, Johnstone EC. A randomized, controlled trial of a brief interventional package for schizophrenic outpatients. Acta Psychiatr Scand 2001; 103: 362-369.

29. Naber D, Vita A. Tools for measuring clinical effectiveness. Eur Neuropsychopharmacol Suppl 2004; 14(4): 435-444.

30. Zygmunt A, Olfson M, Boyer CA, Mechanie D. Interventions to improve medication adherence in schizophrenia. Am J Psychiatry 2002; 159: 1653-1664.

Kati Aho-Mustonen, M.A. (Psychology)

University of Joensuu Department of Psychology P.O. Box 111, FIN-80101 Joensuu FINLAND

Tel. + 358132515396 .

email: kati.aho-mustonen@joensuu.fi 